\section{Giant condyloma acuminate due to human papillomavirus type 16 in an infant successfully treated with topical imiquimod therapy}

\author{
Meltem Dinleyici, ${ }^{1}$ Nurhan Saracoglu, ${ }^{2}$ \\ Makbule Eren, ${ }^{3}$ Ömer Kiliç, ${ }^{4}$ \\ Evrim Ciftci, ${ }^{5}$ Cigdem Sag, ${ }^{6}$ Ates Kara ${ }^{7}$ \\ Ener Cagri Dinleyici, ${ }^{8}$ \\ Deprtments of 'Social Pediatrics, \\ ${ }^{2}$ Dermatology, ${ }^{3}$ Pediatric \\ Gastroenterology and Hepatology, \\ ${ }^{4}$ Department of Pediatric Infectious \\ Disease, ${ }^{5}$ Pathology, Eskisehir Osmangazi \\ University; ${ }^{6}$ Department of Pediatrics, \\ Haydarpasa Numune Training Hospital, \\ Istanbul; ${ }^{7}$ Pediatric Infectious Disease \\ Unit, Hacettepe University, Ankara; \\ ${ }^{8}$ Pediatric Intensive Care Unit and \\ Pediatric Infectious Disease, Eskisehir \\ Osmangazi University, Turkey
}

\begin{abstract}
Anogenital warts related to human papillomavirus (HPV) have been observed in children. Definition of the transmission mode, therapy, and follow-up for long term potential complications is important. A 27-month old girl was admitted with multiple pedunculated redpurple colored cauliflower-like lesions of 1.5 years duration. Clinical/histopathological and microbiological diagnosis was condyloma acuminate due to HPV type 16 . After 12 weeks of imiquimod 5\% cream application (peasized) overnight three times per week, the perianal warts had completely disappeared. The mode of transmission of HPV 16 in our case was probably horizontal, related to the sharing of common personal hygiene items in the women's shelter. We report herein the case of an infant living in a women's shelter with giant condyloma acuminata due to HPV 16, which was successfully treated with topical imiquimod therapy. This patient should be followed up for recurrence and potential malignant lesions related to HPV type 16.
\end{abstract}

\section{Introduction}

Genital warts, including condyloma acuminata, may be observed during childhood. ${ }^{1}$ The number of children with anogenital human papillomavirus (HPV) is unknown; however, recent reports show that an increase in incidence has paralleled the epidemic in adults. ${ }^{2}$
The prevalence of anogenital warts in childhood is $1.5 \%$, and they are observed in girls two times more commonly than in boys. ${ }^{3}$ There is limited data about the incidence of anogenital condyloma acuminate in childhood. ${ }^{4}$ The overall prevalence of infection with specific human papilloma virus types and the true incubation period for children is not well known; however, it is important to define the transmission mode, the therapy, and the follow-up for long term potential complications. ${ }^{5-7}$ Although pediatric genital warts may spontaneously resolve, the treatment of extensive perianal genital warts in children can be challenging. Imiquimod, although not approved for the pediatric population, may be a choice for cases unresponsive to conventional treatment. ${ }^{2,-11}$ Herein, we present the case of a 27-month old girl with condyloma acuminata due to HPV type 16 , which was successfully treated with topical imiquimod therapy.

\section{Case Report}

A 27-month old girl was admitted to our clinic with an enlarging, multiple, perianal lesions of 1.5 years duration. She had been previously treated with topical therapies including cryotherapy but the lesions worsened. Her medical history was unremarkable. The patient had lived in a women's shelter with her mother at the beginning of the lesion. Her mother's medical history and physical examinations were unremarkable for HPV lesions. Physical examination revealed multiple pedunculated red-purple colored cauliflower-like lesions around the anus. There were extensive perianal exophytic lesions. The perianal lesions extended circumferentially around the anus in a width of 3 to $5 \mathrm{~cm}$ (Figure 1A). There was no sign of sexual abuse in her previous records or in our detailed history/physical examination. Histopathological examination demonstrated papillomatosis, acanthosis, and focal koilocytosis. PCR study of the lesion (performing for HPV $6,11,16,18$ ) showed that the cells were positive for HPV type 16, and the clinical/histopathological diagnosis was condyloma acuminata. Topical cryotherapy was performed again; however, the lesions continued to worsen. Therapy with imiquimod $5 \%$ cream was initiated with special permission because this agent was not licensed for condyloma acuminata in our country. Imiquimod 5\% cream (pea-sized amount, half a sachet) was applied to the perianal warts 3 times weekly at night for 6 weeks. After 4 weeks of imiquimod cream therapy, substantial improvement was evident (Figure 1B). Regarding to the improvement of lesions, we continued the imiquimod therapy for 12 weeks. At the end of 12 weeks of therapy, the perianal warts had completely dis-
Correspondence: Meltem Dinleyici, Department of Social Pediatrics, Faculty of Medicine,

Eskisehir Osmangazi University, Eskisehir, TR26480 Turkey.

Tel.: $+90.222 .229 .0064 / 2708$

E-mail: meltemayata@hotmail.com

Key words: Condyloma accuminata; human papillomavirus; HPV type 16; imiquimod; genital warts.

Contributions: MD, NS, ME, OK, EC, AK, ECD, diagnosis; MD, NS, ME, OK, CS, AK, ECD, followup; MD, ME, OK, EC, CS, drafting the article; MD, NS, ME, OK, EC, CS, AK, ECD, final approval of the version to be published.

Conflict of interest: the authors declare no potential conflict of interest.

Received for publication: 2 August 2015.

Revision received: 17 August 2015.

Accepted for publication: 19 August 2015.

This work is licensed under a Creative Commons Attribution NonCommercial 3.0 License (CC BYNC 3.0).

○C Copyright M. Dinleyici et al., 2015

Licensee PAGEPress, Italy

Dermatology Reports 2015; 7:6134

doi:10.4081/dr.2015.6134

appeared. The treatment was very well tolerated with no serious adverse side effects. Minor redness at the site of application was observed. The mode of transmission of HPV 16 in our case was probably horizontal, related to the sharing of common personal hygiene items in the women's shelter.

\section{Discussion}

Human papillomavirus types 6 and 11 account for the majority of cases of genital warts including condyloma acuminata; however, other types of HPV may be seen. Although most HPV infections are subclinical, condyloma acuminata may be a visible manifestation of HPV infection among children less than 4 years old. ${ }^{7,12}$ The mean age at presentation for anogenital warts in children ranges from 2.8 to 5.6 years in a reported case series. ${ }^{7}$ The symptoms of our case had been observed by her mother at 12 months of age. The transmission routes of HPV infection in children are not as clear as in adults. ${ }^{3}$ Proposed mechanisms of perinatal and postnatal transmission include vertical transmission, horizontal transmission (autoinoculation, heteroinoculation), and fomite transmission ${ }^{5-8,13}$ Condyloma acuminata in children may be, but is not always, an indicator of sexual abuse. ${ }^{7}$ Anogenital lesions that are 
noticed in a child before its first birthday are generally acquired perinatally (vertical transmission), either in utero or during vaginal delivery. ${ }^{13}$ However, the origin of lesions that appear between the age of 1 and 3 years old is not as certain. The possibilities include vertical transmission, horizontal transmission, and transmission through sexual abuse. Anogenital warts in children should always raise suspicion of possible sexual abuse, but every case needs to be individually evaluated to determine the level of suspicion. Typically, the probability that sexual abuse is the mode of transmission increases with the age of the child. Vertical transmission is generally accepted to be possible until 12 to 24 months of age. ${ }^{4,8}$ Marcoux et $a l .{ }^{6}$ concluded that under age 3 , a child may become contaminated by heteroinoculation from their caretaker's warts during routine hygienic care and diapering. In $57 \%$ of children with anogenital acuminata, the HPV infection results from non-sexual transmission. ${ }^{4}$ Our case lives in a shelter with her mother and has no history or physical signs of sexual abuse. She was admitted to our clinic at 27 months of age; however, there were no signs of sexual abuse according to her previous medical charts. Her mother also had no complaints associated with HPV infection, and her gynecological examination was normal. We thought that fomites would be the key factor for transmission of HPV infection in the shelter, although perinatally transmission can not be ruled out. Condyloma acuminata in children may be, but is not necessarily, an indicator of sexual abuse. ${ }^{4}$ Therefore, it is difficult to define whether the transmission route in these cases is sexual or not. ${ }^{3,4}$ Although most small children acquire their anogenital warts in innocuous ways, the possibility of sexual abuse must be seriously considered. ${ }^{2}$ A careful medical and social history must be taken to exclude any sign of neglect or abuse, and referral to child protective services is indicated if necessary. In children, anogenital warts are commonly associated with HPV types 6 and 11 . Our patient's condyloma acuminata were related to a high risk HPV type, HPV 16. Unger et al..$^{13}$ evaluated 534 children with genital warts, and they found $2.1 \%$ positive cases to be due to HPV type 16 , and among 14 cases with confirmed sexual abuse, no HPV type 16 was found. The virus type does not provide proof for the presence or absence of sexual abuse in children. ${ }^{10,14}$ Many treatment modalities have been evaluated in the adult population, but the treatment of extensive anogenital warts in children may be more challenging. No available drug therapy effectively eliminates HPV. ${ }^{2}$ Current therapeutic options for the pediatric population such as topical acids, cryotherapy, and surgical excision are often painful, may result in scarring, and may require frequent office or operating room visits. ${ }^{2,11,14}$ Factors that can influence the choice of treatment include the age of the child, the extent and location of the lesions, and the social and family context. Our patient had been treated with topical acids and repeated cryotherapy; however, her lesions worsened. Imiquimod, a topical aminoquinolone immune response modifier, has not been approved for younger children; however, there are reports in the literature of its use in younger children for genital warts. $^{2,8-11}$ It stimulates the production of interferon alpha, tumor necrosis factor, and other cytokines able to inhibit viral replication. Imiquimod 5\% cream is an effective treatment option for children with extensive and rapidly progressive perianal warts. In our case, we applied small amount of imiquimod per treatment in spite of large perianal lesion and we observed clinical improvement without side effects. Gruber and Wilkinson have been also used imiquimod for the treatment of their pediatric cases, and they preferred to use imiquimod application twice weekly. ${ }^{15}$ Avoiding contact with normal skin or decreasing the frequency of imiquimod use, minimizes this risk of local and severe reactions. Imiquimod is usually well tolerated, and the side effects, which include local pain, erythema, pruritus, and irritation, are normally mild and limited to the site of application. ${ }^{10}$ The advantage of imiquimod treatment for children includes the convenience

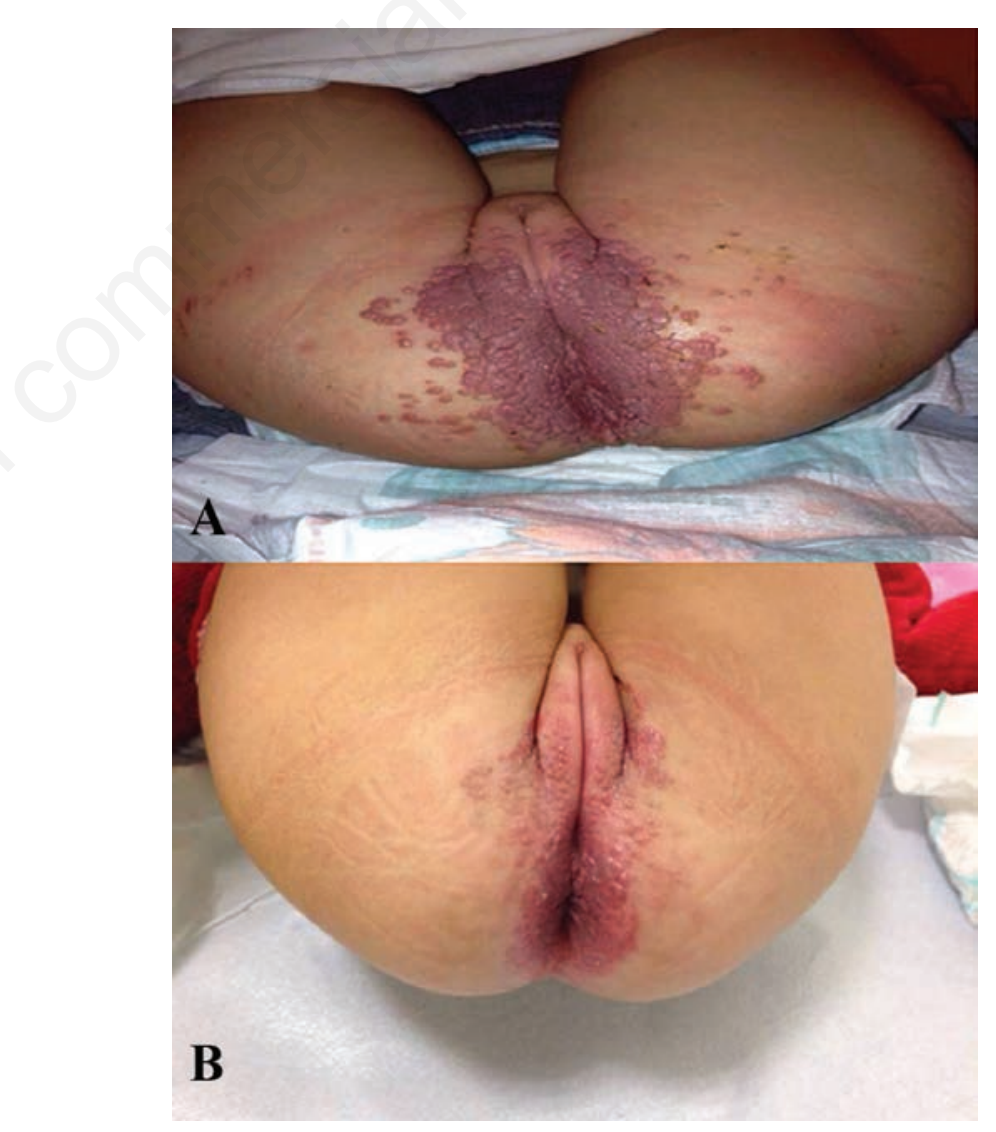

Figure 1. A) Rapidly progressing extensive perianal condyloma in a 3 year old girl; B) Excellent clinical response of perianal condylomata after 4 week use of imiquimod $5 \%$ cream. of a generally well tolerated treatment that can be applied by the child's parent or caregiver, usually painlessly, at home. Its use should be considered in children with extensive condyloma to avoid the pain and possible scarring associated with other approved treatment modalities. Our patient was treated medically with imiquimod 5\% cream, resulting in the complete resolution of her symptoms and lesions. Despite the fact that it is not approved for use in children under the age of 12 , clinicians may wish to consider using imiquimod in a child with extensive genital and perianal condyloma. Imiquimod $5 \%$ cream is a convenient, safe, and effective alternative therapy that should be considered as an option to aggressive, painful, and potentially traumatizing outpatient treatments and also to surgical treatments that can otherwise result in post-operative pain and genital scarring. ${ }^{8}$ Human papilloma virus vaccines are key prevention strategies for anogenital warts and for other clinical conditions related to HPV. In Australia, national population-based hospital data have confirmed previous clinic-based reports of a marked decline in genital warts among adolescents and young adults after the routine use of quadrivalent HPV vaccine. ${ }^{16}$ 


\section{Conclusions}

We can conclude that there are few reports about condyloma acuminata related to HPV type 16 and imiquimod therapy in children. We report herein the case of an infant living in a women's shelter with giant condyloma acuminata due to HPV 16, which was successfully treated with topical imiquimod therapy. This patient should be followed up on a long term basis to guard against recurrence and potential malignant lesions related to HPV type 16.

\section{References}

1. Culton DA, Morrell DS, Burkhart CN. The management of condyloma acuminata in the pediatric population. Pediatr Ann 2009;38:368-72.

2. Campaner AB, Santos RE, Galvão MA, et al. Effectiveness of imiquimod 5\% cream for treatment of extensive anogenital warts in a seven-year-old child. Pediatr Infect Dis J 2007;26:265-6.

3. Bülbül S, Demirceken F, Cakir B, et al. Difficulties in diagnosing sexual abuse in children with condyloma acuminata in Turkey. J Child Sex Abus 2010;19:35-42.

4. Bussen S, Sütterlin M, Schmidt U, Bussen D. Anogenital warts in childhood -always a marker for sexual abuse? Geburtshilfe Frauenheilkd 2012;72:43-8.

5. Jayasinghe Y, Garland SM. Genital warts in children: what do they mean? Arch Dis Child 2006;91:696-700.

6. Marcoux D, Nadeau K, McCuaig C, et al. Pediatric anogenital warts: a 7-year review of children referred to a tertiary-care hospital in Montreal, Canada. Pediatr Dermatol 2006;23:199-207.

7. Stefanaki C, Barkas G, Valari M, et al. Condylomata acuminata in children. Pediatr Infect Dis J 2012;31:422-4.

8. Leclair E, Black A, Fleming N. Imiquimod $5 \%$ cream treatment for rapidly progressive genital condyloma in a 3-year-old girl. J Pediatr Adolesc Gynecol 2012;25:e119-21.

9. Majewski S, Pniewski T, Malejczyk M, Jablonska S. Imiquimod is highly effective for extensive, hyperproliferative condyloma in children. Pediatr Dermatol 2003;20:440-2.

10. Masuko T, Fuchigami T, Inadomi T, et al. Effectiveness of imiquimod 5\% cream for treatment of perianal warts in a 28 -month- old child. Pediatr Int 2011;53:764-6.

11. Stefanaki C, Lagogiani I, Kouris A, et al. Cryotherapy versus imiquimod $5 \%$ cream combined with a keratolytic lotion in cutaneous warts in children: a randomized study. J Dermatolog Treat 2015:1-3.

12. Monk BJ, Tewari KS. The spectrum and clinical sequelae of human papillomavirus infection. Gynecol Oncol 2007;107:S6-13.

13. Unger ER, Fajman NN, Maloney EM, et al. Anogenital human papillomavirus in sexually abused and non-abused children: a multicenter study. Pediatrics 2011;128: e658-65.

14. Altinay-Kirli E, Güçer S, Karnak I. Perianal giant condyloma acuminata in an infant: an alarming lesion for a pediatric surgeon. Turk J Pediatr 2011;53:333-6.

15. Gruber PC, Wilkinson J. Successful treatment of perianal warts in a child with $5 \%$ imiquimod cream. J Dermatolog Treat 2001;12:215-7.

16. Smith MA, Liu B, McIntyre P, et al. Fall in genital warts diagnoses in the general and indigenous Australian population following implementation of a national human papillomavirus vaccination program: analysis of routinely collected national hospital data. J Infect Dis 2015;211:91-9. 